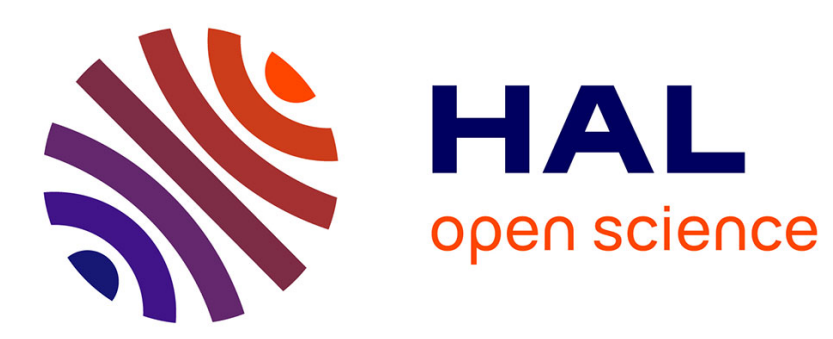

\title{
Thermal motion of a nonlinear localized pattern in a quasi-one-dimensional system
}

Tommy Dessup, Christophe Coste, Michel Saint-Jean

\section{To cite this version:}

Tommy Dessup, Christophe Coste, Michel Saint-Jean. Thermal motion of a nonlinear localized pattern in a quasi-one-dimensional system. Physical Review E , 2016, 94, pp.12217 - 12217. 10.1103/PhysRevE.94.012217 . hal-01404788

\section{HAL Id: hal-01404788 \\ https://hal-univ-paris.archives-ouvertes.fr/hal-01404788}

Submitted on 29 Nov 2016

HAL is a multi-disciplinary open access archive for the deposit and dissemination of scientific research documents, whether they are published or not. The documents may come from teaching and research institutions in France or abroad, or from public or private research centers.
L'archive ouverte pluridisciplinaire HAL, est destinée au dépôt et à la diffusion de documents scientifiques de niveau recherche, publiés ou non, émanant des établissements d'enseignement et de recherche français ou étrangers, des laboratoires publics ou privés. 


\title{
Thermal motion of a nonlinear localized pattern in a quasi-one-dimensional system
}

\author{
Tommy Dessup, Christophe Coste, and Michel Saint Jean \\ Laboratoire Matière et Systèmes Complexes, UMR No. 7057, CNRS, Université Paris 7 Diderot, 75205 Paris Cedex 13, France
}

(Received 21 March 2016; published 15 July 2016)

\begin{abstract}
We study the dynamics of localized nonlinear patterns in a quasi-one-dimensional many-particle system near a subcritical pitchfork bifurcation. The normal form at the bifurcation is given and we show that these patterns can be described as solitary-wave envelopes. They are stable in a large temperature range and can diffuse along the chain of interacting particles. During their displacements the particles are continually redistributed on the envelope. This change of particle location induces a small modulation of the potential energy of the system, with an amplitude that depends on the transverse confinement. At high temperature, this modulation is irrelevant and the thermal motion of the localized patterns displays all the characteristics of a free quasiparticle diffusion with a diffusion coefficient that may be deduced from the normal form. At low temperature, significant physical effects are induced by the modulated potential. In particular, the localized pattern may be trapped at very low temperature. We also exhibit a series of confinement values for which the modulation amplitudes vanishes. For these peculiar confinements, the mean-square displacement of the localized patterns also evidences free-diffusion behavior at low temperature.
\end{abstract}

DOI: 10.1103/PhysRevE.94.012217

\section{INTRODUCTION}

Quasi-one-dimensional systems of interacting particles transversely confined in periodic channels allow nonlinear localized patterns. In this paper we focus on the thermal motion of these patterns, which exhibit surprisingly rich dynamics. These systems of interacting particles are conceptually simple and have been extensively studied for their configurational properties. For instance, laser-cooled ions in Paul traps (the socalled ions crystals or Coulomb crystals) are good candidates to create entangled states, which is a key step toward quantum information [1]; understanding the classical behavior of these systems is a necessity, which motivated several recent works on Coulomb crystals [2-13]. There are many other physical systems consisting of interacting particles confined in narrow channels, with a typical size that extends several orders of magnitude, such as optically confined paramagnetic colloidal particles [14-16], plasma dust in electrostatic traps [17-21], and electrostatically interacting macroscopic beads $[13,22-$ 24].

In these confined systems, the equilibrium positions of the particles result from the competition between the repulsive interaction energy $U(r)$ and the transverse confining potential, which may be approximated by a harmonic potential of stiffness $\beta$. At large stiffness, the particles are aligned along the axis of the confining cell. When $\beta$ decreases just below a critical value $\beta_{Z Z}$, it becomes energetically favorable for the particles to adopt a staggered row configuration. This structural phase transition is known as the zigzag transition [3-7,14-18,20,22,23]. There are two symmetric zigzag patterns with the same energy, so the zigzag transition is basically a pitchfork bifurcation.

The nature of this transition depends on the interaction range and the boundary conditions implied by the geometry of the confining cell. In Refs. $[13,24]$ we have shown that one has to distinguish Coulombic (infinite range) interactions from finite-range interactions and cells with rigid walls at each extremities from cells of annular shape (or cyclic boundary conditions in the longitudinal direction). For
Coulombic interactions, regardless of the boundary conditions, the bifurcation is always supercritical [3,5,6,13]. For cells with rigid extremities, regardless of the interaction range, the bifurcation is also supercritical [14,15,20,21,23]. In contrast, for short-range interactions and cyclic boundary conditions, a subcritical pitchfork bifurcation is evidenced $[13,16,19,24]$. The physical origin of this change in nature of the bifurcation is the nonlinear coupling between the soft mode at the bifurcation and the Goldstone mode due to the translational invariance along the cyclic channel [24].

As a consequence of the subcriticality, there is a range of transverse confinement that allows phases to coexist, corresponding to a localized zigzag pattern surrounded by aligned particles $[19,24,25]$. These localized zigzag patterns are well described as a zigzag phase modulated by a solitarywave envelope [24] (see Fig. 1 for an example) and will henceforth be called bubbles.

These bubbles are equilibrium patterns that are stable in a large temperature range [25]. This is evidenced in Fig. 1, which exhibits the motion of a bubble on a long-time scale (much longer than the typical time scales of the system; see Sec. III A). In this example the temperature is high enough for the bubble displacement to extend up to the simulated cell size and nevertheless the bubble is preserved during the whole simulation. Note that the bubble displacements correspond to the motion of a solitary-wave envelope and not to a global displacement of the particles themselves. Indeed, during the bubble motion, the entire set of particles involved in the localized zigzag pattern is completely renewed for large enough displacements. This will be clearly seen later by a comparison between the trajectory of a typical particle and the trajectory of the bubble itself [see Fig. 5(a)].

Thus, for each bubble envelope position the particles adopt a specific configuration, which will be calculated along with its corresponding potential energy $E_{p}$. The change in particle positions relative to the bubble envelope induces a small variation of the potential energy of the chain, which is a periodic function of the bubble position. We emphasize that this periodic 

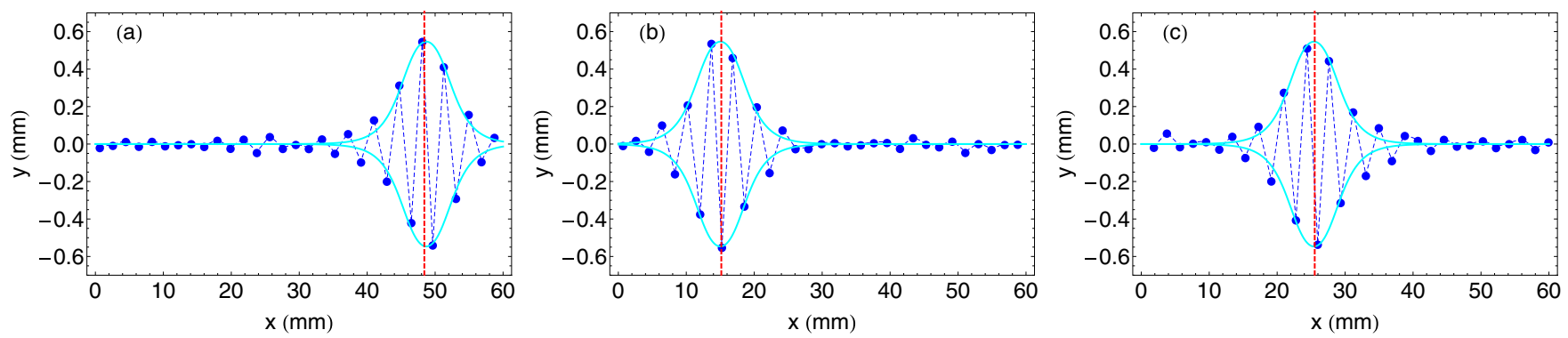

FIG. 1. Three snapshots of a bubble in a system of $N=32$ particles in a cell of length $L=60 \mathrm{~mm}$, for a temperature $T=5 \times 10^{9} \mathrm{~K}$ and $\epsilon=0.08$. The blue circles are the coordinates $\left(x_{i}, y_{i}\right)$ of the point particles at time (a) $100 \mathrm{~s}$, (b) $500 \mathrm{~s}$, and (c) $700 \mathrm{~s}$. The solid cyan line is the analytical bubble shape of Eq. (5). This shape is the same in every plot. The red straight line indicates the bubble position given by Eq. (14).

potential energy is inherently related to the discrete character of the system.

In this paper we show that, at high temperature, the bubbles behave as quasiparticles undergoing free thermal diffusion and we calculate their diffusion coefficients. At low temperature we evidence the sensitivity of the bubble motion to the modulation of the potential energy $E_{p}$. In particular we show that for temperatures low enough for the thermal energy to be of the same order as this potential energy modulation, the bubble motion may be described as that of a quasiparticle in a periodic potential. For lower temperatures the bubble may be trapped by this potential.

The influence of the discrete character of the underlying system on the motion of solitary waves has been already observed in several classical systems. The oldest is the dislocation motion in crystals, with the Frenkel-Kontorova model [26]. Other examples are ferroelectric domain walls, walls between incommensurate phases, soliton dynamics in magnetic materials, and nonlinear dynamics of DNA; for an extensive review see [27]. Note that in all the reported analyses, these effects arise from the solitary-wave motion along an external periodic potential. This is not the case here, where the potential is intrinsic to the discrete system itself.

We recall in Sec. II the theoretical description of the bubbles in the framework of a continuous nonlinear model. We define the mass of the quasiparticle that describes a bubble and calculate the potential energy variation due to the relative displacement of the bubble envelope with respect to the underlying particle chain. The numerical simulations and data analysis are described in Sec. III. The bubble motion exhibited in simulations done at high temperature is reported on in Sec. IV. In Sec. V we focus on low-temperature bubble behavior and discuss the relevant discretization effects. We summarize in Sec. VI. Three Appendixes are devoted to technical details.

\section{THEORETICAL DESCRIPTION OF THE ZIGZAG BUBBLES}

\section{A. Bubbles as solitary-wave envelope}

In this section we summarize the analytic description of a bubble as solitary-wave envelope [24] and calculate its effective mass. Let us consider $N$ particles aligned along the $x$ axis, in a cell of length $L$, in the limits $L \rightarrow \infty$ and $N \rightarrow \infty$ at constant density $N / L \equiv 1 / d$. The repulsive interaction potential between the particles is denoted by $U(r)$ and the stiffness of the transverse confining harmonic potential is denoted by $\beta$. In the vicinity of the zigzag transition, $\beta=\beta_{Z Z}(1-\epsilon)$ with $|\epsilon| \ll 1$, where $\beta_{Z Z}$ is the bifurcation threshold at which the aligned particle configuration becomes unstable. Throughout the paper, the parameter $\epsilon$ measures the transverse confinement and is therefore the control parameter. A particle at the point $\{x, 0\}$ for $\beta \gg \beta_{Z Z}$ is found at the position $\{x+\phi(x, t), \pm h(x, t)\}$ for $\beta \leqslant \beta_{Z Z}$, where the field $\phi(x, t)$ describes the longitudinal modulation of the particle positions, the field $h(x, t)$ describes the absolute value of the particle transverse displacements, and the sign depends on the particle rank. We take into account the interactions up to next nearest neighbors and the fields $\phi(x, t)$ and $h(x, t)$ are assumed to be slowly varying functions of $x$ and of time $t$. We work in dimensionless units, with the distances $x, \phi$, and $h$ expressed in units of $d$ and the time expressed in units of $d / c_{\perp}$, where $c_{\perp}$ is the velocity of the long-wavelength linear transverse waves (see Appendix A).

A systematic expansion of the energy density, in powers of $\epsilon$, shows that the dynamics near the bifurcation is given by the Lagrangian density

$$
\begin{aligned}
\mathcal{L}= & \frac{1}{2}\left(\frac{\partial h}{\partial t}\right)^{2}+\frac{1}{2}\left(\frac{\partial \phi}{\partial t}\right)^{2}+\frac{\epsilon \beta_{Z Z}}{2} h^{2}-\frac{a_{3}}{2}\left(h^{4}+h^{2} \frac{\partial \phi}{\partial x}\right) \\
& -\frac{1}{2}\left(\frac{\partial h}{\partial x}\right)^{2}-\frac{b_{3}}{2}\left(\frac{\partial \phi}{\partial x}\right)^{2}-\frac{a_{5}}{6} h^{6},
\end{aligned}
$$

where all coefficients are expressed as functions of the interaction potential $U(r)$ and its derivatives (see Appendix A). The corresponding field equations are

$$
\begin{gathered}
\frac{\partial^{2} \phi}{\partial t^{2}}=a_{3} h \frac{\partial h}{\partial x}+b_{3} \frac{\partial^{2} \phi}{\partial x^{2}}, \\
\frac{\partial^{2} h}{\partial t^{2}}=\epsilon \beta_{Z Z} h-a_{3}\left(2 h^{3}+h \frac{\partial \phi}{\partial x}\right)+\frac{\partial^{2} h}{\partial x^{2}}-a_{5} h^{5} .
\end{gathered}
$$

We look at the stationary solutions $\{\phi(x), h(x)\}$ of this system. Equation (2) is easily integrated to give

$$
\frac{d \phi}{d x}=-\alpha h(x)^{2} \quad \text { where } \quad \alpha \equiv \frac{a_{3}}{2 b_{3}} .
$$

When the expression (4) is substituted into (3), the coefficient of $h^{3}$ becomes $a_{3}(\alpha-2)$, which ensures a subcritical zigzag transition for $\alpha>2$ that allows phase coexistence. It is therefore consistent to look for localized zigzag patterns, with 
an envelope $h(x)$ that is a stationary solution of Eq. (3) that vanishes at $x \rightarrow \pm \infty$. In Ref. [24] it is found as

$$
h(x)=\frac{h_{+} h_{-}}{\sqrt{\left(h_{+}^{2}-h_{-}^{2}\right) \cosh ^{2}\left(\sqrt{-\epsilon \beta_{Z Z}} x\right)+h_{-}^{2}}},
$$

where

$$
\begin{aligned}
h_{ \pm}^{2}= & \frac{3 a_{3}(\alpha-2)}{4 a_{5}}\left(1 \pm \sqrt{1+\frac{16 \epsilon \beta_{Z Z} a_{5}}{3 a_{3}^{2}(\alpha-2)^{2}}}\right) \text { for } \\
& -\frac{3 a_{3}^{2}(\alpha-2)^{2}}{16 \beta_{Z Z} a_{5}} \leqslant \epsilon \leqslant 0 .
\end{aligned}
$$

The longitudinal modulation $\phi(x)$ associated with the bubble envelope may be deduced from Eq. (4) after integration and reads

$$
\phi(x)=-\alpha \frac{h_{+} h_{-}}{\sqrt{-\epsilon \beta_{Z Z}}} \operatorname{arctanh}\left[\frac{h_{-}}{h_{+}} \tanh \left(\sqrt{-\epsilon \beta_{Z Z}} x\right)\right],
$$

where the integration constant is such that $\phi(x=0)=0$.

The description of the bubble as a solitary-wave envelope also allows one to calculate its effective mass. Indeed, from the Lagrangian density (1), we deduce the stress-energy tensor [28] and calculate the linear momentum density $\mathcal{P}$,

$$
\mathcal{P}=-\frac{\partial \mathcal{L}}{\partial\left(\frac{\partial \phi}{\partial t}\right)} \frac{\partial \phi}{\partial x}-\frac{\partial \mathcal{L}}{\partial\left(\frac{\partial h}{\partial t}\right)} \frac{\partial h}{\partial x}=-\left(\frac{\partial \phi}{\partial t} \frac{\partial \phi}{\partial x}+\frac{\partial h}{\partial t} \frac{\partial h}{\partial x}\right) .
$$

For a bubble that moves at constant velocity $V$ such that $h(x, t)=h(\xi \equiv x-V t)$, the linear momentum density becomes

$$
\mathcal{P}=V\left[\left(\frac{d \phi}{d \xi}\right)^{2}+\left(\frac{d h}{d \xi}\right)^{2}\right]=V\left[\alpha^{2} h^{4}+\left(\frac{d h}{d \xi}\right)^{2}\right],
$$

where we have injected (4) to get the rightmost expression. The linear momentum of the bubble is given by the integration of (9) on the whole $\xi$ axis, with $h(\xi)$ given by (5). We can therefore define the mass of a bubble as $M_{B}=\lim _{V \rightarrow 0} \int_{-\infty}^{\infty}[\mathcal{P}(\xi) / V] d \xi$. After some algebra, we get

$$
\begin{aligned}
M_{\mathrm{B}}= & \frac{3 \alpha^{2} \sqrt{-\epsilon \beta_{Z Z}}}{a_{5}}\left[\frac{1+\chi^{2}}{\chi} \operatorname{arctanh} \chi-1\right] \\
& -\frac{\sqrt{3} \epsilon \beta_{Z Z}}{4 \sqrt{a_{5}}}\left[\frac{1+\chi^{2}}{\chi}-\frac{\left(1-\chi^{2}\right)^{2}}{\chi^{2}} \operatorname{arctanh} \chi\right],
\end{aligned}
$$

where $0<\chi \equiv h_{-} / h_{+}<1$.

Let us add two comments. First, to establish (9), we have used (4), which is valid for stationary bubbles. It is shown in Appendix B that this is still the case for bubbles that move at low velocity $V \ll 1$. Moreover, we show that thermal motion fulfills this inequality, so $M_{\mathrm{B}}$ is a very good estimate of the mass of the bubble as a quasiparticle.

Furthermore, despite the obvious fact that actual systems are always finite, the analysis performed in the thermodynamic limit remains relevant to experiments in annular cells $[19,29]$ or to simulations with cyclic boundary conditions [24]. Indeed, the rotational invariance exhibited by such systems ensures the existence of a Goldstone mode, hence the subcriticality of the bifurcation. In order to apply our continuous model to a finite cyclic system, we have to consider an effective infinite system with a set of parameters $\epsilon_{\text {eff }}$ and $d_{\text {eff }}$ that are properly calculated to ensure that the length of the particle chain is equal to the actual length $L$ of the cyclic cell (see Ref. [24] and Appendix C). The constants in Eqs. (2) and (3) are then evaluated for $d=d_{\text {eff }}$ and $\epsilon=\epsilon_{\text {eff }}$. From now on, for the sake of simplicity, the subscript "eff" will be omitted. In Fig. 1 we plot the bubble envelope $h\left(x-x_{0}\right)$, where $x_{0}$ represents the position of the envelope apex. The functional form $h(\cdot)$ of the bubble is uniquely determined by the system size and the parameter $\epsilon$ used in the simulations: Once the effective distance is deduced from Eq. (C2), the bubble envelope follows from Eq. (5). The position $x_{0}$ is thus the only fitting parameter in the plots of Fig. 1. Therefore, Fig. 1 represents snapshots of the same bubble and evidences that thermal fluctuations induce the motion of the bubble in the simulation cell, without deformations.

\section{B. Effects of the discrete character of the system}

Beyond this continuous description, the system consists of a finite number $N$ of particles. In consequence, an actual bubble is a configuration of particles distributed according to the continuous solitary wave $\{\phi(x), h(x)\}$ described previously. Each configuration is determined by the relative position of the envelope apex with respect to the underlying chain. Let us first put a system of $N$ equidistant particles on an envelope with the apex exactly located on the longitudinal position of the particle $i_{0}$. The corresponding discrete configuration is

$$
\begin{aligned}
& x_{i}=d\left[i+\phi\left(i-i_{0}\right)\right], \\
& y_{i}=d(-1)^{i} h\left(i-i_{0}\right)
\end{aligned}
$$

for $i \in[1, N]$. In the case where the envelope apex is located between two neighboring particles, the chain of particles is shifted by $\Delta$ with respect to the envelope. The particle positions then become

$$
\begin{aligned}
& x_{i}=d\left[i+\Delta+\phi\left(i-i_{0}+\Delta\right)\right], \\
& y_{i}=d(-1)^{i} h\left(i-i_{0}+\Delta\right)
\end{aligned}
$$

for $i \in[1, N]$. These configurations are thus periodic with a period $\Delta=1$. Two examples of such configurations are shown in Fig. 2(a) for $\Delta=0$ and Fig. 2(b) for $\Delta=1 / 2$.

Note that the symmetry of the configuration depends on the envelope position. By construction, the first configuration $(\Delta=0)$ exhibits a mirror symmetry relative to the plan $x=i_{0} d$. Since $h(x)$ is an even function [see Eq. (5)] and $\phi(x)$ is an odd function [see Eq. (7)], this symmetry happens more generally when the shift is $\Delta=p$, with $p$ an integer. The second configuration $(\Delta=1 / 2)$ has an inversion symmetry with respect to the center $\left\{x=\left(i_{0}-1 / 2\right) d, y=0\right\}$. In the general case, the center of this inversion symmetry is $\left\{x=\left(i_{0}-p-1 / 2\right) d, y=0\right\}$ when $\Delta=p+1 / 2$, with $p$ an integer.

Knowing the particle positions as a function of the envelope displacement $\Delta$ [see Eq. (12)], we can compute the potential energy $E_{p}(\epsilon, \Delta)$ of the corresponding configuration. This energy $E_{p}(\epsilon, \Delta)$ is plotted in Fig. 3 as a function of $\Delta$ for two values of the confinement $\epsilon$ that correspond to localized patterns of different widths. As a consequence of 

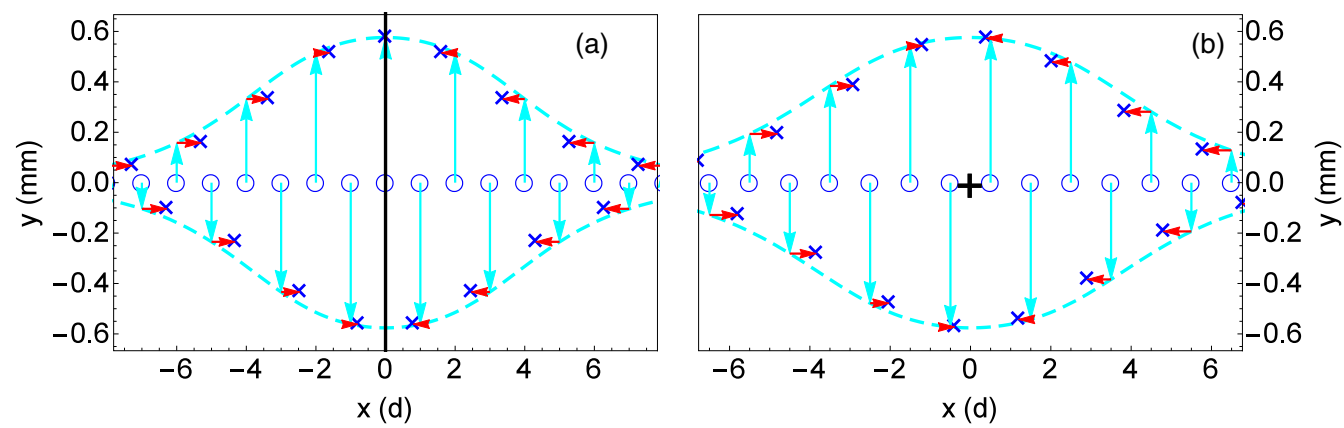

FIG. 2. Discrete configurations of the particles in a bubble, zoomed in on the center particles. The number of particles is $N=64$ and the cell length is $L=120 \mathrm{~mm}$. The abscissa is the longitudinal position in units of $d$ and the ordinate is the transverse positions in mm. The dashed cyan line is the solitary-wave envelope (5) centered at $x=0$. The open circles indicate the unperturbed chain of particles and the crosses are the actual positions of the particles in the bubble. The arrows show the displacements of the particles. (a) The center particle is at the bubble apex $\Delta=0$ [see Eq. (11)]. The solid black line indicates the plane of mirror symmetry. (b) Configuration shifted by half a period $\Delta=1 / 2$ [see Eq. (12)]. The thick black plus sign indicates the symmetry center.

the periodicity of the discrete configurations, this potential energy is necessarily a periodic function of $\Delta$ with period 1 . We have found that $E_{p}(\epsilon, \Delta)$ is a sinusoidal function of $\Delta$,

$$
E_{p}(\epsilon, \Delta)=\frac{\Delta E(\epsilon)}{2} \cos 2 \pi \Delta .
$$

Surprisingly, the configurations associated with an extremum of potential energy depend on the confinement. In the case of Fig. 3(a) the configuration of minimum energy corresponds to a particle located at the envelope apex $(\Delta=p$, with $p$ an integer), whereas in the case of Fig. 3(b) the configuration of minimum energy corresponds to the envelope apex located at the middle of two particles $(\Delta=p+1 / 2$, with $p$ an integer).

Moreover, a systematic study of the amplitude $\Delta E(\epsilon)$ shows that it varies with the confinement parameter $\epsilon$ as a succession of arches between zero and a maximum that slowly increases with $\epsilon$ (see Fig. 4). These arches are almost regularly spaced, the difference between two successive zeros of $\Delta E(\epsilon)$ being roughly constant [see Fig. 4(b)].
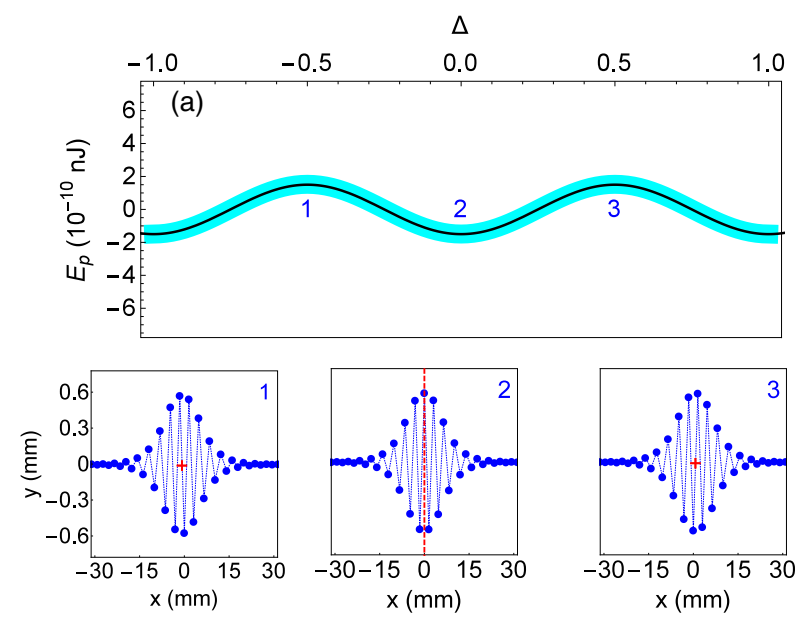

For a given arch, the respective symmetries of the configurations associated with the minimum and the maximum of $E_{p}(\epsilon, \Delta)$ are fixed, whereas for the next arch these symmetries are reversed. The blue dashed lines in Fig. 4(a) correspond to confinements for which the minimal energy configurations exhibit a mirror symmetry. For instance, the blue square in Fig. 4(a) corresponds to the energy plotted in Fig. 3(a). The solid red lines in Fig. 4(a) correspond to confinements for which the minimal energy configurations exhibit a center symmetry. The red circle in Fig. 4(a) corresponds to the energy plotted in Fig. 3(b).

\section{SIMULATIONS AND DATA ANALYSIS}

\section{A. Simulated system}

We simulate the dynamics of $N$ identical point particles of mass $m$ moving on a plane, submitted to a thermal bath at temperature $T$ by the numerical integration of coupled Langevin equations [30]. The thermal bath is accounted for

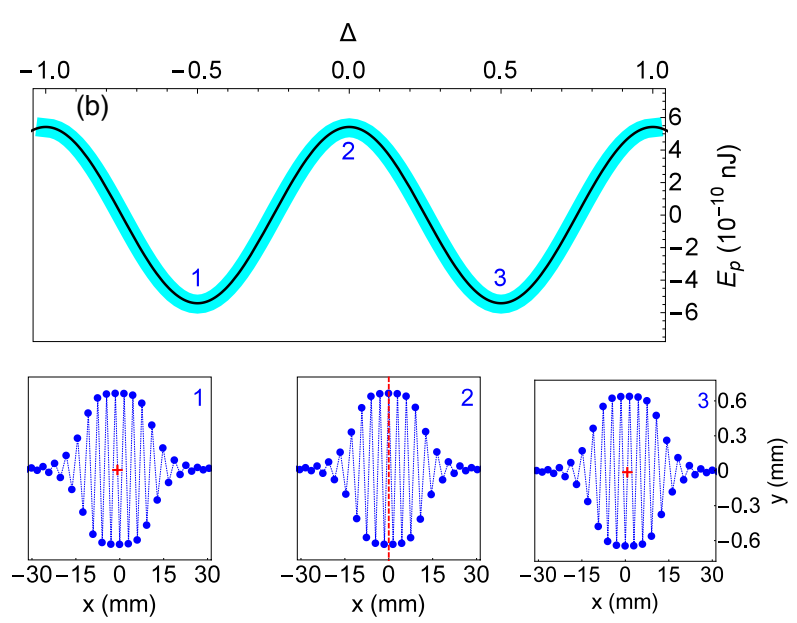

FIG. 3. (a) and (b) The thick solid cyan line plots the potential energy for a bubble configuration $\left(10^{-10} \mathrm{~nJ}\right)$ as a function of the dimensionless shift $\Delta$, for $N=64$ particles in a cell of length $L=120 \mathrm{~mm}$ and for confinement (a) $\epsilon=0.077$ and (b) $\epsilon=0.22$. The thin black solid line is a sinusoidal fit. Below we plot the particle configurations that correspond to the indices 1, 2, and 3 in (a) and (b). The dashed red line indicates the mirror symmetry plane and the red plus sign indicates the center of symmetry. 

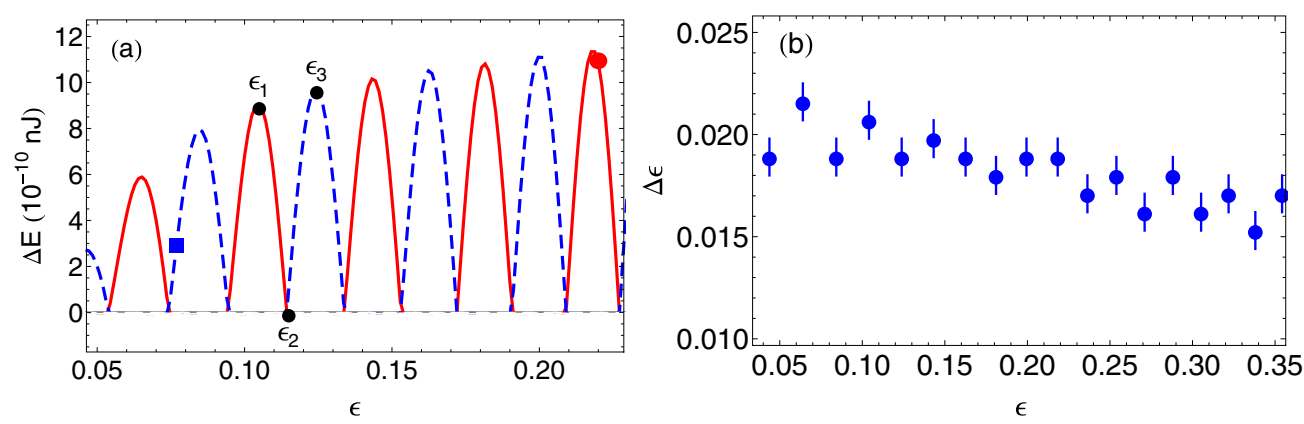

FIG. 4. (a) Plot of the energetic barrier $\Delta E(\epsilon)$ (in $10^{-10} \mathrm{~nJ}$ ) as a function of the confinement $\epsilon$, for $N=64$ particles in a cell of length $L=120 \mathrm{~mm}$. The dashed blue line corresponds to confinements for which the configurations of minimal energy exhibit a mirror symmetry and the solid red line corresponds to confinements for which the configurations of minimal energy exhibit a center symmetry. The peculiar confinements $\epsilon_{1}, \epsilon_{2}$, and $\epsilon_{3}$ are discussed in Sec. V. The blue square indicates the confinement of Fig. 3(a) and the red dot indicates the confinement of Fig. 3(b). (b) Plot of the difference between two successive zeros of $\Delta E(\epsilon)$ as a function of the confinement $\epsilon$.

by a damping constant $\gamma$ and by random forces applied on each particle, with the statistical properties of uncorrelated white Gaussian noise. The length of the simulation cell is $L$. The particles interact with a repulsive potential, which in all simulations is a screened electrostatic interaction $U(r)=$ $U_{0} K_{0}\left(r / \lambda_{0}\right)$ with energy scale $U_{0}$ and a characteristic length $\lambda_{0} \ll L$, where $K_{0}$ is the modified Bessel function of index 0 (see [30]). The particles are transversally confined in a quasi-one-dimensional geometry by a harmonic potential of stiffness $\beta$ and periodic boundary conditions are applied in the longitudinal direction. In all simulations we take $\lambda_{0}=$ $0.48 \mathrm{~mm}$ and $d \equiv L / N=1.875 \mathrm{~mm}$, which ensures $\alpha>2$ in Eq. (4), hence a subcritical pitchfork bifurcation.

The temperature scale is fixed by the choice of $U_{0}$. Somewhat arbitrarily, we have chosen the relevant energy scale of an experimental system of charged macroscopic beads [29,31], so $U(d) \approx 0.117 \mathrm{~nJ}$ or $U(d) / k_{B} \approx 8.46 \times 10^{12} \mathrm{~K}$. Above $T \sim 5 \times 10^{12} \mathrm{~K}$, the homogeneous zigzag pattern is destroyed by the thermal fluctuations. We have shown in Ref. [25] that the stability domain of the bubbles toward thermal fluctuations extends up to $10^{10} \mathrm{~K}$. Thermal motion of the bubbles is seen in the simulations down to $10^{6} \mathrm{~K}$, as shown in Sec. V. The temperature range that we consider in this paper is thus extended to four orders of magnitude, between $10^{6}$ and $10^{10} \mathrm{~K}$.

The bubble motion is studied for damping constants $\gamma=1$ and $10 \mathrm{~s}^{-1}$. This is to be compared to the duration of the simulation runs, which is typically $10^{5} \mathrm{~s}$, and to the typical time scales $d / c \sim 0.1 \mathrm{~s}$ and $L / c \sim 10 \mathrm{~s}$ (for $L=60 \mathrm{~mm}$ ), where $c$ is the typical velocity of acoustic waves in the system (see Appendix A).

An important feature of our analysis is the dependence of the transport coefficients on the shape of the bubble envelope. We therefore vary the system size $N$ and the transverse confinement $\beta$ in order to change the shape of the bubbles. The range of transverse confinement that allows the appearance of bubbles in the simulations has been given elsewhere (see Fig. 3 of Ref. [24]). We have restricted these domains somewhat because the consistency of the bubble mass calculation requires a bubble size that is much smaller than $L$. When expressed in terms of the dimensionless parameter $\epsilon$, the parameters used in this paper are $\epsilon \in[0.130,0.340]$ for $N=16, \epsilon \in$ $[0.060,0.400]$ for $N=32$, and $\epsilon \in[0.004,0.130]$ for $N=64$.

\section{B. Measurements of the bubble position}

In order to study the bubble motion, we could obtain its instantaneous position from a fit by the analytic expression of the solitary-wave envelope, as shown by the solid cyan line in Fig. 1. However, the numerical process to adjust the position of the solitary-wave envelope is time consuming. Thus we use a much quicker method, based on the instantaneous particle positions $\left\{x_{i}(t), y_{i}(t)\right\}$, which enhances the weight of the particles inside the bubble [large $\left.\left|y_{i}(t)\right|\right]$. The bubble position $x_{\mathrm{B}}(t)$ is defined as

$$
x_{\mathrm{B}}(t)=\frac{\sum_{i=1}^{N} x_{i}(t) y_{i}{ }^{2}(t)}{\sum_{i=1}^{N} y_{i}{ }^{2}(t)} .
$$

The validity of this method has been verified by comparison with the lengthier one discussed previously, as shown in Fig. 1.

A typical trajectory $x_{\mathrm{B}}(t)$ is shown in Fig. 5(a), together with the longitudinal motion of a typical particle. The bubble motion extends along many interparticle distances. During the same time, a typical particle evidences much smaller longitudinal displacements. This confirms that the bubble motions is not associated with global longitudinal motion of the particles, but with the motion of the solitary-wave envelope leading to a transverse reorganization of the particles.

The trajectory $x_{\mathrm{B}}(t)$ fluctuates on two very different time scales, presenting some fast fluctuations of small amplitude embedded in a slower displacement of much larger amplitude. These two time scales correspond to different dynamical processes. The slowest motion results from the motion of the bubble envelope and will mainly be described in the next section. In contrast, the fast fluctuations are associated with thermal excitations of the individual particles or of their collective vibrational modes. These fast transverse fluctuations induce local rearrangement of the particles, which results in jumps of the bubble of a typical distance $d$. This is made clear in Fig. 5(b), where we compare the bubble position $x_{\mathrm{B}}(t)$ with the position $x_{i}(t)$ of the particle with maximum transverse displacement $\left|y_{i}(t)\right|$.

\section{BUBBLE FREE DIFFUSION AT HIGH TEMPERATURE}

In this section we consider the bubble motion that takes place when the temperature is much larger than the amplitude 

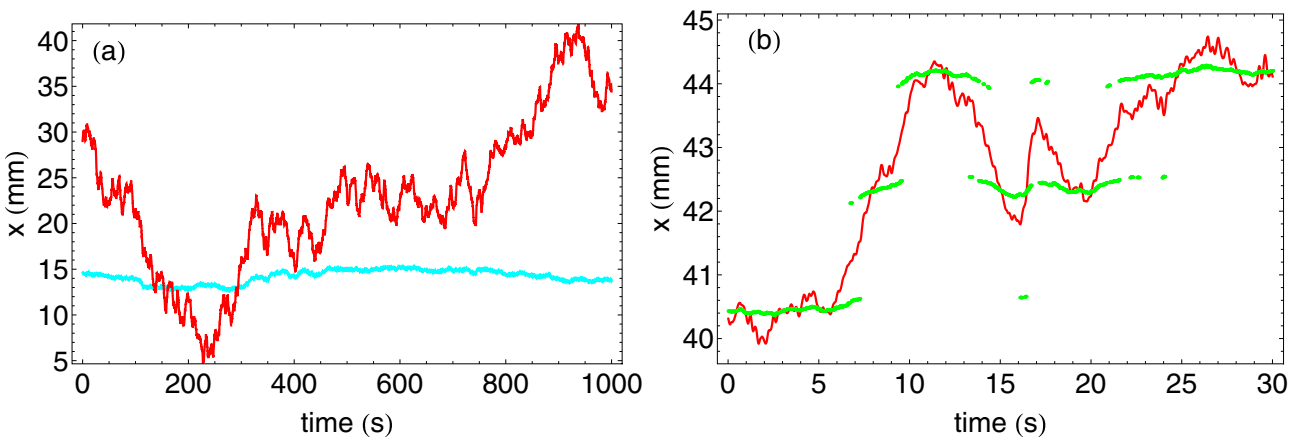

FIG. 5. Plot of the longitudinal displacements (in $\mathrm{mm}$ ) as a function of time (in s), for a system of $N=32$ particles, $L=60 \mathrm{~mm}$, at $T=5 . \times 10^{9} \mathrm{~K}, \gamma=1 \mathrm{~s}^{-1}$, and $\epsilon=0.08$. (a) Typical trajectory of a bubble, measured by $x_{\mathrm{B}}(t)$ [red (dark gray) solid line], and longitudinal motion of a typical particle [cyan (light gray) solid line]. (b) Zoom in on the bubble trajectory (red solid line) [note the scale difference from (a)] and instantaneous position of the particle with maximum transverse displacement (green dots).

$\Delta E(\epsilon)$ of the periodic potential due to the discreteness of the system, discussed in Sec. II B.

\section{A. Bubble mean-square displacement}

As shown in Fig. 5, the bubble motion looks like a random walk. We therefore consider the bubble trajectory statistical properties and calculate the mean-square displacement (MSD) $\left\langle\Delta x_{\mathrm{B}}^{2}(t)\right\rangle$. In Fig. 6 we show the bubble MSD at three temperatures $T=10^{9}, 5 \times 10^{9}$, and $10^{10} \mathrm{~K}$ for two dissipation coefficients $\gamma=1$ and $10 \mathrm{~s}^{-1}$. In Figs. 6(a) and 6(c) we also display, for the sake of comparison, the longitudinal MSD of a typical particle in the chain, which is two orders of magnitude smaller. When we plot $\left\langle\Delta x_{\mathrm{B}}{ }^{2}(t)\right\rangle / k_{B} T$, as in Figs. 6(b) and $6(d)$, we see a collapse of the data, which evidences that the bubble behaves as a coherent pattern that undergoes stochastic motion because of a thermal bath. The plots of Fig. 6 extend over six time decades and clearly exhibit two asymptotic regimes. At very short times (in Fig. $6, t \leqslant 0.1 \mathrm{~s}$ ) the bubble MSD scales as $t^{2}$ and is independent of $\gamma$. In contrast, at long times (in Fig. $6, t \geqslant 10 \mathrm{~s}$ ) the bubble MSD scales as $t$ with a coefficient that decreases as $1 / \gamma$ as the friction increases.

Note that the characteristic times of the two asymptotic regimes for the bubble motion might be estimated from the
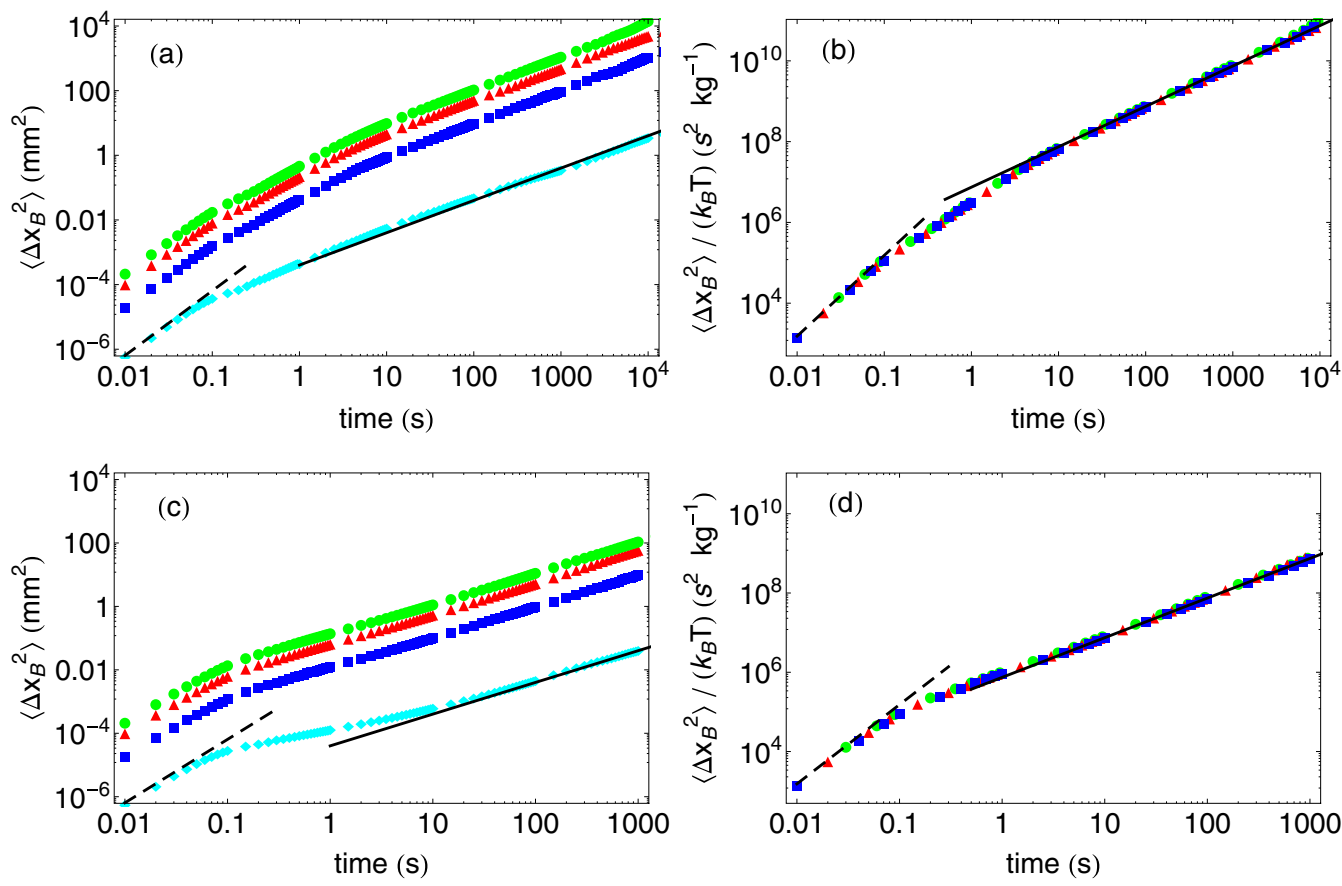

FIG. 6. (a) and (c) Plots of the bubble MSD (logarithmic scale, in $\mathrm{mm}^{2}$ ) as a function of time (logarithmic scale, in s) for $N=32$ particles in a cell of length $L=60 \mathrm{~mm}$ and $\epsilon=0.08$. The temperatures are $T=10^{9} \mathrm{~K}$ (blue squares), $T=5 \times 10^{9} \mathrm{~K}$ (red triangles), and $T=10^{10} \mathrm{~K}$ (green circles). The damping constant is (a) $\gamma=1 \mathrm{~s}^{-1}$ and (c) $\gamma=10 \mathrm{~s}^{-1}$. For the sake of comparison, the longitudinal MSDs of a typical particle in the system with temperature $T=10^{9} \mathrm{~K}$ are shown as cyan diamonds. The dashed black lines correspond to the ballistic regime and the solid black lines to the diffusive regimes for a single particle in the chain [30]. (b) and (d) Plots of $\left\langle\Delta x_{\mathrm{B}}^{2}(t)\right\rangle / k_{B} T$ (logarithmic scale, in $\mathrm{s}^{2} \mathrm{~kg}^{-1}$ ) as a function of time (logarithmic scale, in s) for (b) $\gamma=1 \mathrm{~s}^{-1}$ and (d) $\gamma=10 \mathrm{~s}^{-1}$. The solid black line is the free diffusion of a bubble of mass $M_{\mathrm{B}}$ defined in Eq. (10) and the dashed black line is the ballistic regime of Eq. (15), without any fitting parameter. 


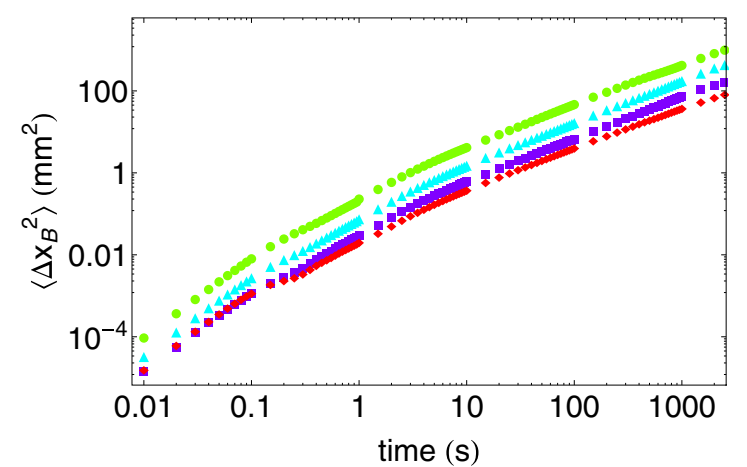

FIG. 7. Plot of the bubble MSD (logarithmic scale, in $\mathrm{mm}^{2}$ ) as a function of time (logarithmic scale, in s) for $\epsilon=0.06$ (green circles), $\epsilon=0.07$ (cyan triangles), $\epsilon=0.10$ (purple squares), and $\epsilon=0.18$ (red diamonds); $N=32$ particles; $L=60 \mathrm{~mm}$; temperature $T=$ $5 \times 10^{9} \mathrm{~K}$; and $\gamma=1 \mathrm{~s}^{-1}$.

typical acoustic wave velocity $c$ in the chain (see Appendix A), the interparticle distance $d$, and the system size $L$. For instance, the characteristic time for a wave to travel between nearest neighbors is $d / c$. It defines the time scale beneath which the particles are independent, so the bubble dynamics results from the uncorrelated fluctuations of individual particles. A rough estimate gives $d / c \sim 0.1 \mathrm{~s}$, which is consistent with the shorttime scale in Fig. 6. On the other hand, the characteristic time $L / c$ for a wave to travel along the whole cell defines the time scale above which long-range correlations occur between the particles, characterizing a regime of coherent displacements of the particles associated with large solitary-wave motion. A rough estimate gives $L / c \sim 10 \mathrm{~s}$, which is again consistent with the long-time scale in Fig. 6.

\section{B. Long-time bubble free diffusion}

As of now, we have some evidence that at long times the bubble behaves like a quasiparticle freely diffusing in a thermal bath. We expect a specific diffusion coefficient $D_{\mathrm{B}}=k_{B} T / M \gamma$, where the mass $M$ is exactly the mass $M_{B}$ calculated in the framework of the continuous model [see Sec. II and Eq. (10)]. In order to check our interpretation, we have measured the bubble MSD for several values of the transverse stiffness by varying the parameter $\epsilon$. These measurements are shown in Fig. 7. A diffusive behavior at long times, with $\left\langle\Delta x_{\mathrm{B}}{ }^{2}\right\rangle \propto t$, is observed regardless of the transverse confinement. The confinement is found to slightly shift the MSD with a diffusion coefficient that decreases with the confinement, which suggests an increase of the quasiparticle mass.

In Fig. 8 we have reported the values of effective diffusion coefficients $D_{\mathrm{B}}$ extracted from the long-time behavior of the bubble MSD for several confinements $\epsilon$ and for three system sizes $N=16,32$, and 64 at a fixed density (see Sec. III A). We see that $D_{\mathrm{B}}(\epsilon)$ is a strongly decreasing function of $\epsilon$ for a given system size. Regardless of the system size, the thin bubbles (small $\epsilon$ ) diffuse much faster than the wide bubbles (large $\epsilon$ ). In order to discriminate if this effect is due to the release of the transverse confinement or if it is a characteristic of the bubble shape, we have taken advantage of

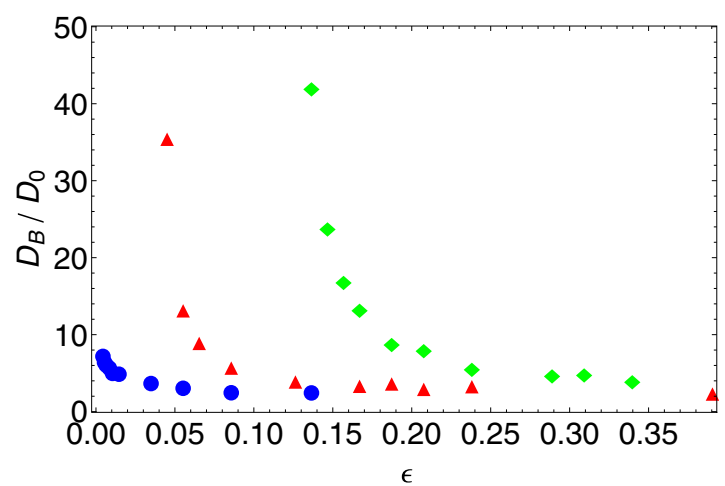

FIG. 8. Plot of the diffusion coefficient $D_{\mathrm{B}}$ extracted from the long-time behavior of the bubble MSD, normalized by the diffusion coefficient of a free single particle $D_{0}=k_{B} T / m \gamma$, as a function of the confinement $\epsilon$. We show data for $N=16$ and $L=30 \mathrm{~mm}$ (green diamonds), $N=32$ and $L=60 \mathrm{~mm}$ (red triangles), and $N=64$ and $L=120 \mathrm{~mm}$ (blue circles).

the possibility to observe an identical solitary-wave envelope at different values of confinement $\epsilon$ with a careful choice of the parameters $(N, \epsilon)$. We show in Fig. 9(a) three bubbles with identical envelopes, observed in systems of $N=64,32$, and 16 particles. The corresponding bubble MSDs measured at the same temperature $T$ and for the same dissipation coefficient $\gamma$ are identical [see Fig. 9(b)]. This evidences that the dependence of the diffusion coefficient on $\epsilon$ is linked to the bubble envelope and not directly to the transverse confinement itself.

We have thus evidenced that, at long times, the bubbles behave as free quasiparticles in a thermal bath and their MSDs only depend on their shape. Therefore, their diffusion coefficient must be $D_{\mathrm{B}}=k_{B} T / M_{\mathrm{B}} \gamma$, where $M_{\mathrm{B}}$ is the mass of a bubble [see Eq. (10)]. In Fig. 10(a) we compare the diffusion coefficients measured from the long-time behavior of the bubble MSD (closed symbols) to the diffusion coefficients $k_{B} T / M_{\mathrm{B}} \gamma$, for two system sizes. ${ }^{1}$ There is no fitting parameter and the agreement is excellent. Moreover, we show in Fig. 10(b) that the diffusion coefficients measured for both system sizes collapse on the same curve and actually scale as the inverse of the effective mass $M_{B}(\epsilon)$.

Note also that the diffusion coefficients are consistent with the picture of a random walk exhibited Fig. 5(b). We may consider that the bubble does random jumps of amplitude $d$ (the interparticle distance) with a characteristic time $\tau$, which would give a diffusion coefficient $D \sim d^{2} / 2 \tau$. For instance, Fig. 5(b) gives a rough estimate of $\tau \sim 5 \mathrm{~s}$; since $T=5 \times$ $10^{9} \mathrm{~K}$ and $\gamma=1 \mathrm{~s}^{-1}$, this gives $D_{\mathrm{B}} / D_{0} \sim 12$, which is of the same order of magnitude as the data displayed in Figs. 7 and 10.

In conclusion, we have shown that the bubble motion at long times may be described as the free diffusion, at temperature $T$, of a quasiparticle of mass $M_{\mathrm{B}}$.

\footnotetext{
${ }^{1}$ As explained in Appendix $\mathrm{C}$, the continuous model makes sense if the system size is much larger than the bubble width. The calculations are valid for $N=32$ and 64 , but systems of only $N=16$ particles are too small. As shown in Fig. 8, the phenomenology is nevertheless the same.
} 


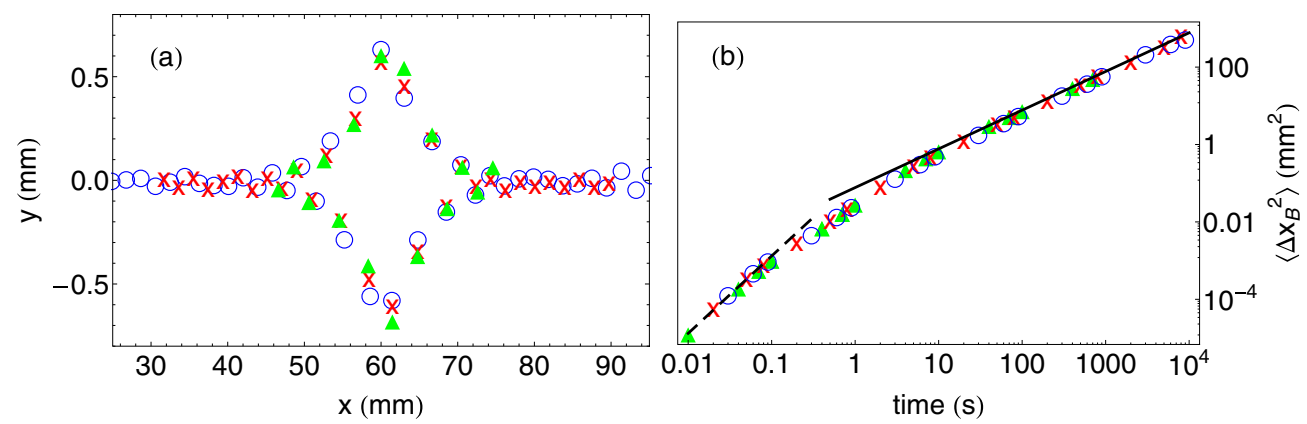

FIG. 9. (a) Three identical bubbles obtained for three sets of parameters $(N, \epsilon)$ : $(16,0.25)$ and $L=30 \mathrm{~mm}$, green triangles; $(32,0.1)$ and $L=60 \mathrm{~mm}$, red crosses; and $(64,0.03)$ and $L=120 \mathrm{~mm}$, blue circles. We display the configurations $x_{i}$ and $y_{i}$ (both with length in mm), with the bubble apex in the middle of the cell. (b) Plot of the bubble MSD (logarithmic scale, in $\mathrm{mm}^{2}$ ) as a function of time (logarithmic scale, in s) for the three configurations of (a), using the same symbols. The temperature is $T=5 \times 10^{9} \mathrm{~K}$ and the dissipation is $\gamma=1 \mathrm{~s}^{-1}$ in every simulation. The solid black line is the free diffusion of a bubble of mass $M_{\mathrm{B}}$ defined in Eq. (10) and the dashed black line is the ballistic regime of Eq. (15), without any fitting parameter.

\section{Short-time bubble behavior}

At short time, for each particle $i$ the positions $x_{i}(t)$ and $y_{i}(t)$ are basically independent random variables. This is the basis for a simple calculation of the $\operatorname{MSD}\left\langle\Delta x_{\mathrm{B}}^{2}(t)\right\rangle$. The particles randomly fluctuate around their equilibrium positions $\bar{x}_{i}$ and $\bar{y}_{i}$. We thus set $x_{i}(t)=\bar{x}_{i}+\delta x_{i}(t)$ and $y_{i}(t)=\bar{y}_{i}+\delta y_{i}(t)$, where the small fluctuations $\delta x_{i}(t)$ and $\delta y_{i}(t)$ are such that $\left\langle\delta x_{i}^{2}(t)\right\rangle=\left(k_{B} T / m\right) t^{2}$ and $\left\langle\delta y_{i}^{2}(t)\right\rangle=\left(k_{B} T / m\right) t^{2}$. Since all the correlations between particle displacements are negligible, by substituting these expressions into Eq. (14) we obtain

$$
\begin{aligned}
\left\langle\Delta x_{\mathrm{B}}^{2}(t)\right\rangle & =\left(\frac{\sum_{i=1}^{N} 4\left(\bar{x}_{i}-\bar{x}_{\mathrm{B}}\right)^{2} \bar{y}_{i}^{2}+\sum_{i=1}^{N} \bar{y}_{i}^{4}}{\left(\sum_{i=1}^{N} \bar{y}_{i}^{2}\right)^{2}}\right) \frac{k_{B} T}{m} t^{2} \\
\text { for } \overline{x_{\mathrm{B}}} & =\frac{\sum_{i=1}^{N} \overline{x_{i}}{\overline{y_{i}}}^{2}}{\sum_{i=1}^{N} \bar{y}_{i}^{2}}
\end{aligned}
$$

As expected, we recover that $\left\langle\Delta x_{\mathrm{B}}^{2}(t)\right\rangle$ scales as $t^{2}$ and is proportional to the temperature and independent of $\gamma$. This explains the collapse evidenced in Figs. 6(b) and 6(d). Moreover, we get an expression for the prefactor of the $t^{2}$ scaling law that may be compared to our measurements. This is done in Figs. 6(b), 6(d), and 9(b), where it is shown, without any fitting parameter, that Eq. (15) describes indeed very well the short-time (typically $t \leqslant 0.1 \mathrm{~s}$ ) behavior of the bubble MSD. Let us emphasize that this $t^{2}$ scaling does not correspond to the ballistic regime of the quasiparticle, which would rather give $\left(k_{B} T / M_{B}\right) t^{2}$, which numerically is ruled out by the data. This is consistent with our assumption that the short-time behavior of the bubble only results from independent fluctuations of the particles.

It is indicated that, when the bubble diffuses with strong dissipation, there exists an intermediate regime between the ballistic and the long-time one for which the MSD is proportional to $t^{\alpha}$ with $\alpha<1$ [see Fig. 6(c)]. This behavior is characterized by the collective modes of vibration characteristic in systems of interacting particles, as we have shown in Ref. [30].

\section{BUBBLE TRAPPING AND ACTIVATED JUMPS AT LOW TEMPERATURE}

The low-temperature regime is reached when the thermal energy $k_{B} T$ is smaller than or of the same order as the

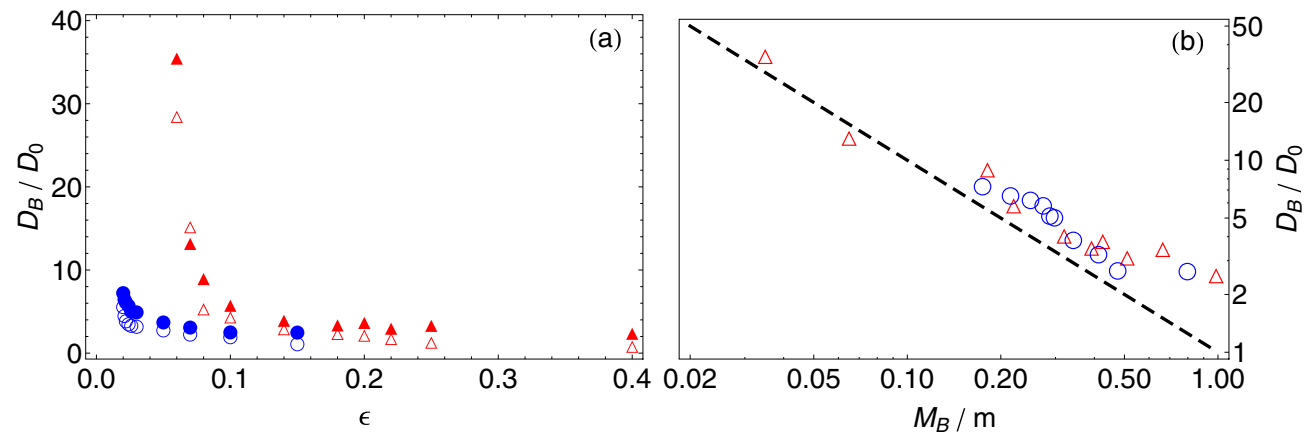

FIG. 10. (a) Plot of the diffusion coefficient $D_{\mathrm{B}}$, normalized by the free particle diffusion coefficient $D_{0}=k_{B} T / m \gamma$, as a function of the confinement $\epsilon$, for $N=32$ and $L=60 \mathrm{~mm}$ (red triangles) and $N=64$ and $L=120 \mathrm{~mm}$ (blue circles). The closed symbols correspond to the diffusion coefficients deduced from the long-time MSD and the open symbols correspond to the calculated diffusion coefficients $k_{B} T / M_{\mathrm{B}} \gamma$, where the mass $M_{B}$ is given by (10). (b) A log-log plot of $D_{\mathrm{B}} / D_{0}$ as a function of the dimensionless mass $M_{\mathrm{B}} / m$ of the quasiparticle. The dashed line is the theoretical expectation of slope -1 . 

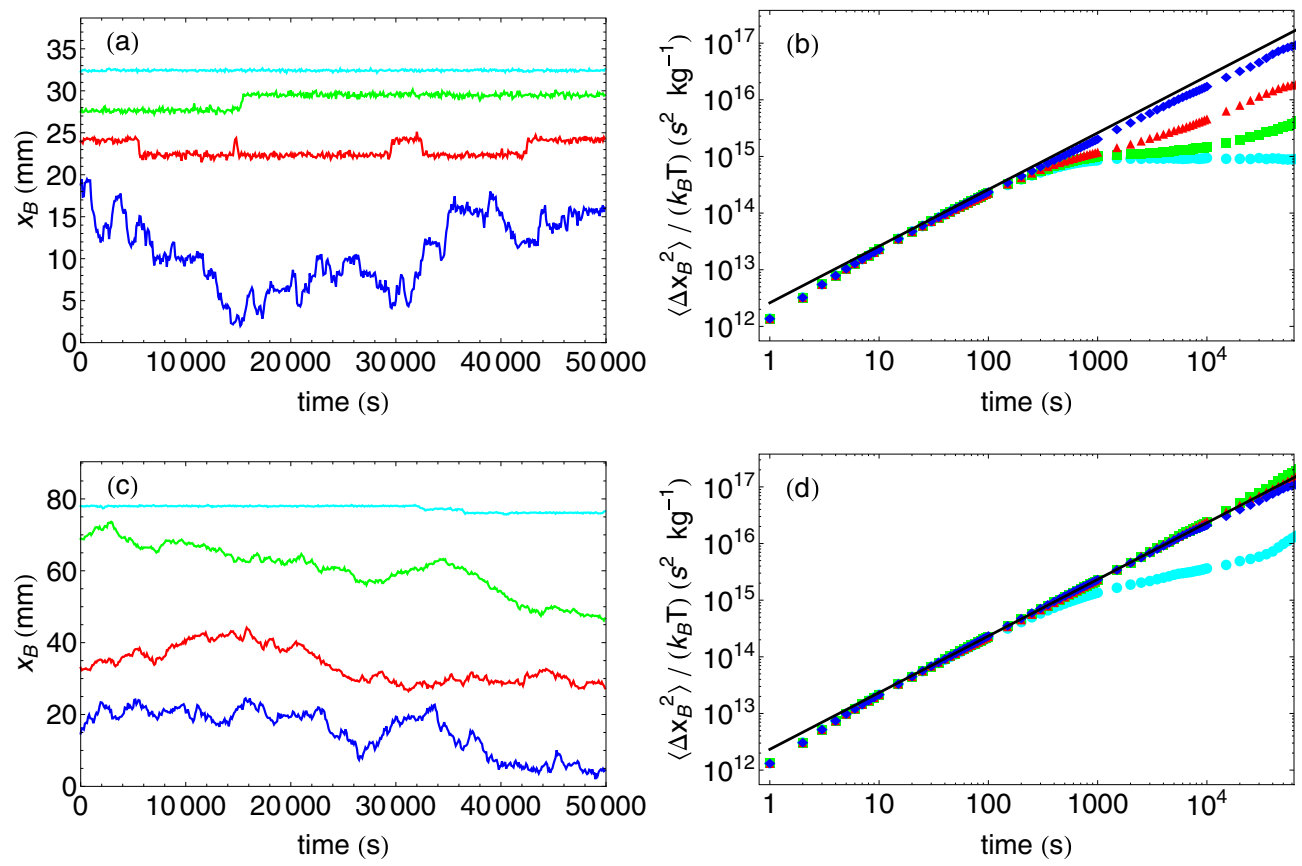

FIG. 11. (a) and (c) Plots of the longitudinal bubble position $x_{\mathrm{B}}$ (in $\mathrm{mm}$ ) as a function of time (in s), for a system of $N=64$ particles in a cell of $L=120 \mathrm{~mm}$ with $\gamma=1 \mathrm{~s}^{-1}$. (a) and (b) The confinement is $\epsilon_{t}=0.085$ and $\Delta E\left(\epsilon_{t}\right)=7.9 \times 10^{-10} \mathrm{~nJ}$. (c) and (d) The confinement is $\epsilon_{b}=0.093$ and $\Delta E\left(\epsilon_{b}\right)=1.010^{-10} \mathrm{~nJ}$. The temperatures are $T=10^{6} \mathrm{~K}$ (cyan circles), $T=5 \times 10^{6} \mathrm{~K}$ (green squares), $T=7 \times 10^{6} \mathrm{~K}$

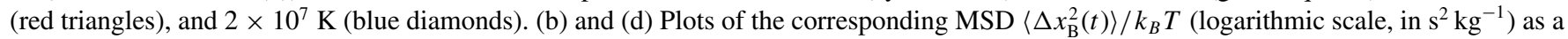
function of time (logarithmic scale, in s).

amplitude of the modulated potential energy. To be specific, we deduce from Figs. 3 and 4 that $\Delta E(\epsilon) \sim 10^{-10} \mathrm{~nJ}$, hence the temperature $T \sim 10^{4} \mathrm{~K}$, which corresponds to a very low temperature in our system (the relevant energy scales are recalled in Sec III A). In this regime, the bubble motion becomes sensitive to the energetic cost induced by the modulated potential $E_{p}(\epsilon, \Delta)$.

\section{A. Trapping and activated jumps}

In the framework of our quasiparticle description, the bubble dynamics may be interpreted as a thermally activated motion in a modulated potential $E_{p}(\epsilon, \Delta)$ [see Eq. (13)]. The motion of a single particle in an external periodic potential has been widely studied [32-34]. When $\Delta E(\epsilon) \gg k_{B} T$, the particle is trapped in a well, leading to a saturation in its MSD. By contrast, when $\Delta E(\epsilon)$ and $k_{B} T$ are comparable, the thermal fluctuations of the particle may allow its motion above the energetic barrier $\Delta E(\epsilon)$ and large motion results from successive jumps from one well to the next one, the characteristic time between two jumps decreasing exponentially with the temperature [33]. Such activated motion results in a smaller diffusion coefficient than for the free particle diffusion [32].

This phenomenology is evidenced by the low-temperature bubble motion. In Fig. 11(a) we display the bubble trajectories for increasing temperatures inducing four different bubble dynamics. For $T=10^{6} \mathrm{~K}$, the bubble is trapped around one potential minimum, its trajectory being roughly a constant. For higher temperatures, we observe discrete bubble jumps between successive potential minima. For $T=5 \times 10^{6} \mathrm{~K}$ only one jump is observed in the time sequence, while for
$T=7 \times 10^{6} \mathrm{~K}$ several jumps occur. These observations agree with the behaviors expected from the quasiparticle model in this intermediate range of temperature for which discrete jumps occur, with a characteristic time between jumps that quickly decreases with the temperature. Eventually, for $T=$ $2 \times 10^{7} \mathrm{~K}$ the temperature is high enough for the bubble not to be trapped anymore, so its trajectory looks like that of a free particle. The corresponding bubble MSD $\left\langle\Delta x_{\mathrm{B}}^{2}(t)\right\rangle$ is plotted in Fig. 11(b). For the lowest temperature $\left(T=10^{6} \mathrm{~K}\right)$ the MSD saturates since the bubble is trapped. In contrast, for intermediate temperatures $\left(T=5 \times 10^{6}\right.$ and $\left.7 \times 10^{6} \mathrm{~K}\right)$, the MSD scales as $t$ at long times after an intermediate regime corresponding to the transitory bubble trapping. The duration of this intermediate regime, as the characteristic time between jumps, decreases with increasing temperature. Eventually, for larger temperature $\left(T=2 \times 10^{7} \mathrm{~K}\right)$ the MSD scales as $t$ as that of a free particle, with the diffusion coefficient $D_{\mathrm{B}}=k_{B} T / M_{B} \gamma$ calculated in Sec. IV, as shown by the black line in Fig. 11(b).

All these behaviors are in perfect qualitative agreement with a description of the bubble motion as that of a quasiparticle of mass $M_{B}$ in an external periodic potential. However, the confinement $\epsilon$ in Fig. 11(a) corresponds to a temperature of $T \sim 7.7 \times 10^{4} \mathrm{~K}$. It thus seems that the calculation of the modulated potential energy described in Sec. II B underestimates the modulation amplitude.

\section{B. Restored free diffusion for specific transverse confinements}

Besides the numerical value of the modulated potential energy, we have evidenced an impressive behavior of the 

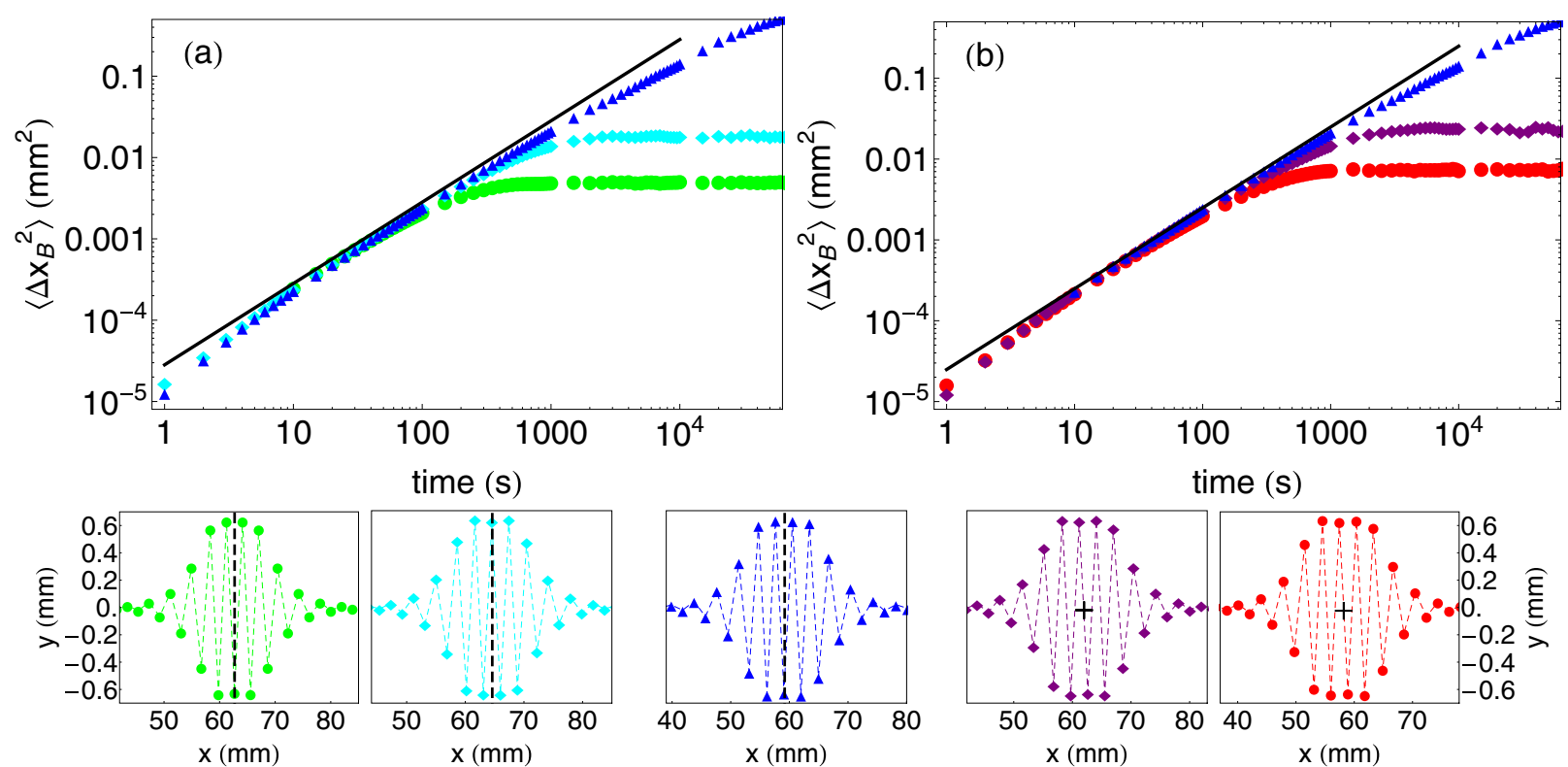

FIG. 12. Plots of the bubble MSD $\left\langle\Delta x_{\mathrm{B}}^{2}(t)\right\rangle$ (logarithmic scale, in $\mathrm{mm}^{2}$ ) as a function of time (logarithmic scale, in s) for a system of $N=64$ particles in a cell of length $L=120 \mathrm{~mm}$ in a thermal bath at $T=10^{6} \mathrm{~K}$ and $\gamma=1 \mathrm{~s}^{-1}$ for the confinements (a) $\epsilon_{1}=0.12$ (green circles), $\epsilon=0.13$ (cyan diamonds), and $\epsilon_{2}=0.134$ (blue triangles) and (b) $\epsilon_{2}=0.134$ (blue triangles), $\epsilon=0.138$ (purple diamonds), and $\epsilon_{3}=0.145$ (red circles). The solid black line is the long-time diffusive behavior with $D_{\mathrm{B}}=k_{B} T / M_{\mathrm{B}} \gamma$. Below we display the minimum energy configurations associated with each confinement, in increasing order of $\epsilon$ from left to right. The dashed lines indicate the mirror symmetry plane and the plus signs indicate the centers of symmetry.

modulation amplitude, which vanishes for specific values of the transverse confinement (see Fig. 4). In order to establish the relevance of our description of the low-temperature bubble motion, we now focus on these specific values $\epsilon^{*}$ of the transverse confinement such that $\Delta E\left(\epsilon^{*}\right)=0$.

In the plots of Figs. 11(c) and 11(d), we display simulations data obtained for such a confinement, with a very small barrier $\Delta E(\epsilon)$, at the same temperatures as for Figs. 11(a) and 11(b). For $T=5 \times 10^{6}, 7 \times 10^{6}$, and $2 \times 10^{7} \mathrm{~K}$ the trajectories are very similar and look like those of freely diffusing particles. This is confirmed by the MSD plots, which show that $\left\langle\Delta x_{\mathrm{B}}^{2}(t)\right\rangle / k_{B} T$ does not depend on the temperature [see Fig. 11(d)]. For the lowest temperature, in contrast with the case of Fig. 11(a), several jumps occur during the simulation, which evidences that the bubble is no longer trapped in a potential well. The bubble MSD is neither that of a freely diffusing quasiparticle nor that of a trapped one.

Let us now focus on the neighborhood of a particular zero of $\Delta E(\epsilon)\left[\epsilon=\epsilon_{2}\right.$ in Fig. 4(a)], considering confinements in the range $\epsilon_{1}<\epsilon<\epsilon_{2}$ and then $\epsilon_{2}<\epsilon<\epsilon_{3}$ in order to explore the whole amplitude $\Delta E(\epsilon)$ between a maximum $\left(\epsilon_{1}\right.$ or $\left.\epsilon_{3}\right)$ and a zero $\left(\epsilon_{2}\right)$. For this small confinement range the bubble envelopes are expected to be very similar and to have almost the same bubble mass $M_{B}$. The corresponding $\operatorname{MSD}\left\langle\Delta x_{\mathrm{B}}^{2}(t)\right\rangle$ is displayed in Fig. 12. As in the previous case, for confinement $\epsilon=\epsilon_{2}$ such that $\Delta E\left(\epsilon_{2}\right) \approx 0$ the MSD only scales as $t$, whereas for the other confinement values for which $\Delta E(\epsilon)>0$ the MSD saturates. The saturation values of the MSD and the times at which they are reached increase as $\epsilon$ goes from $\epsilon_{1}$ up to $\epsilon_{2}$, which is consistent with the fact that $\Delta E(\epsilon)<\Delta E\left(\epsilon_{1}\right)$ [see Fig. 12(a)]. In Fig. 12(b) the saturation values of the
MSD and the times at which they are reached decrease as $\epsilon$ goes from $\epsilon_{2}$ up to $\epsilon_{3}$, which is again consistent with the fact that $\Delta E(\epsilon)<\Delta E\left(\epsilon_{3}\right)$. Before saturation the MSDs behave as $D_{B} t$, with the same coefficient $D_{B}=k_{B} T / M_{B} \gamma$ whatever $\epsilon$ is, which is consistent with the fact that the bubble envelopes are very much the same, as shown by the bottom plots Fig. 12. Note that, as expected from Figs. 3 and 4, the symmetry of the bubble configuration changes when the confinement crosses the zero of $\Delta E(\epsilon)$. The configurations displayed in Fig. 12 show for $\epsilon_{1}<\epsilon<\epsilon_{2}$ a mirror symmetry, whereas for $\epsilon_{2}<\epsilon<\epsilon_{3}$ they present an inversion point symmetry.

This analysis has been extended to the whole $\epsilon$ range of Fig. 4 by a systematic study of the bubble MSD saturation values $\left\langle\Delta x_{\mathrm{B}}^{2}\right\rangle_{\text {sat }}$ and the configuration symmetries. The results are presented in Fig. 13. We observe a series of regularly spaced peaks of the bubble MSD saturation value. These peaks should be associated with very small values of $\Delta E(\epsilon)$. In Table I we display these peak locations deduced from Fig. 13 together with the zeros of $\Delta E(\epsilon)$ (see Fig. 4). We see that both sets of numerical values are very much the same. Moreover, the alternate change of configuration symmetry predicted in Fig. 4 is indeed observed, as indicated in Fig. 13.

The values of $\Delta E(\epsilon)$ can be estimated from the saturation values of the bubble MSD. For a particle trapped in a minimum of the potential $E_{p}(\epsilon, \Delta)$ [see Eq. (13)], the saturation value is expected to be $\left\langle\Delta x^{2}\right\rangle_{\text {sat }}=k_{B} T d^{2} / 4 \pi^{2} \Delta E$. The estimated $\Delta E(\epsilon)$ are plotted Fig. 13(b). The resulting plot is in qualitative agreement with Fig. 4, but the measured energetic barriers are two orders of magnitude higher than the one expected. Despite this discrepancy in the numerical values of the energetic barrier, we have provided in this section convincing evidence that 
(a)

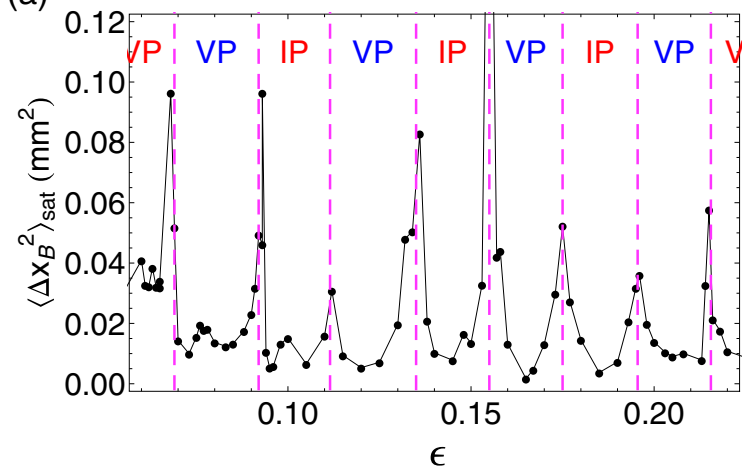

(b)

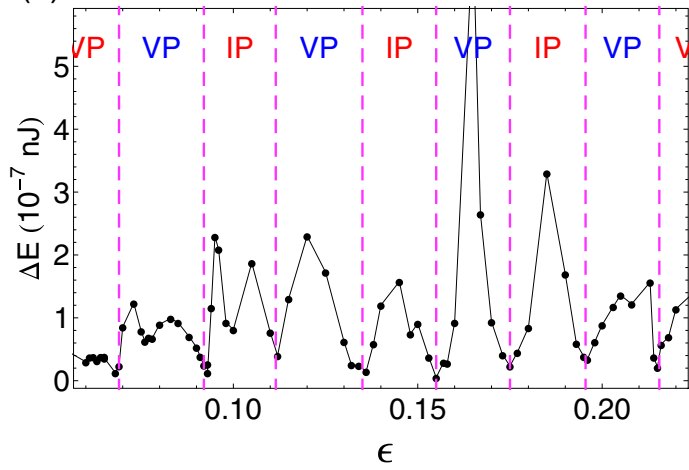

FIG. 13. (a) Plot of the saturation values of the $\operatorname{MSD}\left\langle\Delta x_{\mathrm{B}}^{2}(t)\right\rangle\left(\right.$ in $\left.\mathrm{mm}^{2}\right)$ as a function of the confinement $\epsilon$, for $N=64$ particles in a cell of $L=120 \mathrm{~mm}$. (b) Plot of the amplitude $\Delta E(\epsilon)$ (in $10^{-7} \mathrm{~nJ}$ ) as a function of the confinement $\epsilon$ (see the text for the calculation method). In both plots the symmetries of the minimal energy configuration are, respectively, indicated in blue for the vertical plan symmetry (VP) and in red for the inversion point symmetry (IP).

the bubble motion at low temperature is very well described like those of a quasiparticle of mass $M_{B}$ in an external periodic potential. This periodic potential is due to the positions of the discrete particles along the solitary-wave envelope. Indeed, for the specific transverse confinement values at which the amplitude of the periodic potential vanishes, the bubble MSD is very strongly enhanced.

\section{CONCLUSION}

In this paper we focused on the dynamics of localized nonlinear patterns in a quasi-one-dimensional system of interacting particles near a subcritical pitchfork bifurcation. These patterns, which we call bubbles, are well described by a solitary-wave envelope deduced from the normal form at the bifurcation.

The bubbles are stable in a large temperature range and diffuse over large distances without any distortion. We describe their thermal motion by their mean-square displacements.

At high temperature, the thermal motion of the bubble displays all characteristics of free quasiparticle diffusion. The relevant diffusion coefficient is given by $D_{B}=k_{B} T / M_{B} \gamma$, where $M_{B}$ is the bubble mass that is analytically deduced from the normal form.

TABLE I. Column 1 lists the zeros of $\Delta E(\epsilon)$ [see Fig. 4 (a)], column 2 the locations of the peaks in the saturation values of the bubble MSD [see Fig. 13(a)], and column 3 the estimate of the error on the peak locations, deduced from their widths.

\begin{tabular}{lcc}
\hline \hline$\epsilon$ & $\epsilon_{\text {num }}$ & $\delta \epsilon_{\text {num }}$ \\
\hline 0.075 & 0.068 & \pm 0.003 \\
0.094 & 0.093 & \pm 0.001 \\
0.114 & 0.112 & \pm 0.003 \\
0.133 & 0.136 & \pm 0.002 \\
0.153 & 0.155 & \pm 0.002 \\
0.172 & 0.175 & \pm 0.002 \\
0.190 & 0.196 & \pm 0.002 \\
0.216 & 0.215 & \pm 0.001 \\
\hline \hline
\end{tabular}

Because of the discrete character of the system, for each bubble envelope position the particles adopt a specific configuration. When we compute the potential energy of the system, we exhibit a periodic modulation induced by the change in particle positions relative to the bubble envelope. The amplitude of this modulation depends on the transverse confinement and vanishes for a series of regularly spaced values.

If these potential energy modulations are irrelevant at high temperature, we have exhibited significant physical consequences at low temperature. For nonzero modulation amplitude, at very low temperature, the bubbles are trapped in the potential minima and their MSD saturates. At higher temperature the bubbles diffuse by random jumps from one minimum to another, so the MSD scales as $t$ at long times after an intermediate regime corresponding to a temporary bubble trapping. For the specific confinement values such that the modulation amplitude vanishes, the bubble exhibits free particle diffusion even at these very low temperatures. The zeros of the modulation amplitude deduced from the simulation data are in very good agreement with the calculation of the discrete configuration energy. From the analysis of the variations of the saturation values of the MSD as a function of the confinement $\epsilon$ we have estimated the amplitude of the modulated potential. Its dependence upon the confinement is in qualitative agreement with the model, but the numerical values are larger than expected.

\section{APPENDIX A: COEFFICIENTS OF THE NONLINEAR MODEL}

In this appendix we express the coefficients in Eqs. (1)-(3) as functions of the interaction potential $U(r)$. We recall that the model includes interactions up to second neighbors only. We also emphasize that all coefficients are dimensionless, hence there are minor differences from the notation of Ref. [24].

The velocities $c_{\perp}$ of transverse acoustic waves and $c_{\|}$of longitudinal waves are

$m c_{\perp}^{2}=d\left[2 U^{\prime}(2 d)-U^{\prime}(d)\right], \quad m c_{\|}^{2}=d^{2}\left[U^{\prime \prime}(d)+4 U^{\prime \prime}(2 d)\right]$, 
where $m$ is the mass of a particle and $U^{\prime} \equiv d U / d r$. The coefficients in Eqs. (2) and (3) are expressed as

$$
\beta_{Z Z}=\frac{-4 d U^{\prime}}{m c_{\perp}^{2}}, \quad a_{3}=\frac{4 d\left(d U^{\prime \prime}-U^{\prime}\right)}{m c_{\perp}^{2}}, \quad b_{3}=\left(\frac{c_{\|}}{c_{\perp}}\right)^{2},
$$

where the derivatives of the potential are to be taken at $r=d$. The coefficients $\beta_{Z Z}, a_{3}$, and $b_{3}$ are positive.

The remaining coefficient is

$$
a_{5}=\frac{2 d}{m c_{\perp}^{2}}\left[2\left(6-6 \alpha+\alpha^{2}\right)\left(U^{\prime}-d U^{\prime \prime}\right)+(2-\alpha)^{2} d^{2} U^{\prime \prime \prime}\right] .
$$

Note that this definition of $a_{5}$ assumes the result of Eq. (4). Using it in (1) or in (3) is thus not rigorous. As said in Ref. [24], we neglect in the Lagrangian density some highly derived terms in $h$ and $\phi$. We have checked that their contributions to the energy of a bubble are negligible. In the remaining terms, we use (4) to get the $h^{6}$ term in the Lagrangian density. The approximation made in the definition of $a_{5}$ is thus physically consistent for stationary patterns.

\section{APPENDIX B: BUBBLES MOVING AT CONSTANT VELOCITY}

A word of caution may be useful about the extension of our calculations to nonstationary bubbles moving at constant velocity $V$. Let $\xi=x-V t$ and let us assume that $h(x, t)=$ $h(\xi)$ and $\phi(x, t)=\phi(\xi)$. Substituting these Ansätze into (2), we deduce

$\left(b_{3}-V^{2}\right) \phi^{\prime \prime}=-a_{3} h h^{\prime} \Longrightarrow \phi^{\prime}=-\frac{\alpha b_{3}}{b_{3}-V^{2}} h^{2} \equiv-\widetilde{\alpha}(V) h^{2}$,

where $h^{\prime}=d h / d \xi$. For subsonic bubbles, $V<1$ (we recall that $c_{\perp}$ is the velocity unit and $\left.b_{3} \approx 4\right)$, hence $\widetilde{\alpha}>\alpha$ so that the subcriticality of the bifurcation is preserved. Therefore, the calculations may proceed exactly as before and we obtain for the amplitude of the moving bubble the same as in (5), but the constant $\alpha$ has to be replaced by $\widetilde{\alpha}(V)$.

In the case of the thermal diffusion of a bubble, the velocity $V$ may be estimated from the bubble mass $M_{B}$ [see Eq. (10)] and the thermal energy, as $V \sim \sqrt{2 k_{B} T / M_{B}}$. We deduce from Fig. 10 that a typical value for the bubble mass is $M_{B} / m \sim$ 0.2 . Thus, even at $T=10^{10} \mathrm{~K}$, we get $V \sim 0.1 \mathrm{~cm} / \mathrm{s}$. In contrast, for an interparticle distance $d=60 / 32 \mathrm{~mm}$, using Eq. (A1) and the numerical values of Sec. III A, we get $c_{\perp} \sim c_{\|} / 2 \sim 1.5 \mathrm{~cm} / \mathrm{s}$. The correction $\left(V / c_{\perp}\right)^{2}$ is thus very small, so $\widetilde{\alpha}(V) \approx \alpha$ is an excellent approximation. Therefore, the stationary bubble given in Eqs. (5) and (6) is an accurate description of nonstationary bubbles that undergo thermal motion.

\section{APPENDIX C: CYCLIC BOUNDARY CONDITIONS}

The analysis of Sec. II A assumes a system in the thermodynamic limit. The simulations are done with $N$ particles in a cell of finite length $L$, with cyclic boundary conditions. These boundary conditions ensure rotational invariance of the simulated system, which preserves the nonlinear soft mode coupling at the basis of Eqs. (2) and (3).

In the simulations, the bubbles are observed when the stiffness $\beta_{\text {simu }}$ of the confining potential is such that $\beta_{\text {simu }}<$ $-4 U^{\prime}(d) / d$, where $d=L / N$ is the mean interparticle distance (note that $\beta_{\text {simu }}$ is assumed to be in $\mathrm{N} / \mathrm{m}$ in this appendix). This defines a positive parameter $\epsilon_{\text {simu }}$ as $\beta_{\text {simu }}=\left[-4 U^{\prime}(d) / d\right](1-$ $\left.\epsilon_{\text {simu }}\right)$. The parameter $\epsilon_{\text {simu }}$ is simply written $\epsilon$ throughout the text. It measures the transverse confining potential taken in a given simulation.

The bubbles are energetically allowed because the longitudinal distance between the particles in the bubble (those with $h>0$ ) is smaller than $d$, so the distance between the particles outside the bubble (those with $h=0$ ) is greater than $d$. A bubble takes place in the simulation cell when the energy increase due to the particles that climb the transverse potential is more than compensated for by the energy loss of the remaining particles that become further apart. Let $d_{\text {eff }}>d$ be the distance between neighboring particles outside the bubble. We may define an effective bifurcation parameter $\epsilon_{\text {eff }}$ such that

$$
\beta_{\text {simu }}=-4 \frac{U^{\prime}(d)}{d}\left(1-\epsilon_{\text {simu }}\right)=-4 \frac{U^{\prime}\left(d_{\mathrm{eff}}\right)}{d_{\mathrm{eff}}}\left(1-\epsilon_{\mathrm{eff}}\right) .
$$

A bubble may appear if this effective bifurcation parameter $\epsilon_{\text {eff }}$ is negative, as shown by Eq. (5). Since $-U^{\prime}(d) / d$ is a strongly decreasing function of the distance, this is possible for $d_{\text {eff }}>d$.

When there is a bubble in the system, the longitudinal distance between neighboring particles is modulated by the variation of $\phi$. The system length is obtained after integrating on the cell range $-L / 2 \leqslant x \leqslant L / 2$. This provides the consistency condition

$$
L=d_{\mathrm{eff}}[N+2 \phi(L / 2)],
$$

since $\phi(x)$ is an odd function [see Eq. (7)]. Here all parameters are known functions of $d_{\text {eff }}$ [see Eqs. (6) and (7) and Appendix A], so the only unknown quantity in this equation is $d_{\mathrm{eff}}$.

In Ref. [25] we have plotted the calculated values of $d_{\text {eff }}$ and the relevant values of $\epsilon_{\text {eff }}{ }^{2}$ For practical purposes both formulas are equivalent. The values of $d_{\text {eff }}$ are always greater than $d$ and the values of $\epsilon_{\text {eff }}$ are negative, which is consistent with our description of the bubbles. A bubble observed in a finite cell of length $L$ is thus interpreted as a bubble in an infinite system with an interparticle distance $d_{\text {eff }}$ at infinity. The calculation of $d_{\text {eff }}$ ensures that the actual length of the simulated cell is preserved. The comparison between the bubble patterns given by the simulations and the analytic expression (5), calculated for the value of $d_{\text {eff }}$ in a way that is described above, exhibits very good agreement (see Fig. 1 in the present paper; other examples are provided in $[24,25])$.

\footnotetext{
${ }^{2}$ Strictly speaking, we have used in Ref. [25] the approximate formula $L=d_{\mathrm{eff}}[N+2 \phi(\infty)]$ rather than Eq. (C2).
} 
[1] R. Blatt and D. Wineland, Entangled states of trapped atomic ions, Nature (London) 453, 1008 (2008).

[2] I. Waki, S. Kassner, G. Birkl, and H. Walther, Observation of Ordered Structures of Laser-Cooled Ions in a Quadrupole Storage Ring, Phys. Rev. Lett. 68, 2007 (1992).

[3] D. G. Enzer, M. M. Schauer, J. J. Gomez, M. S. Gulley, M. H. Holzscheiter, P. G. Kwiat, S. K. Lamoreaux, C. G. Peterson, V. D. Sandberg, D. Tupa, A. G. White, R. J. Hughes, and D. F. V. James, Observation of Power-Law Scaling for Phase Transitions in Linear Trapped Ion Crystals, Phys. Rev. Lett. 85, 2466 (2000)

[4] S. Seidelin, J. Chiaverini, R. Reichle, J. J. Bollinger, D. Leibfried, J. Britton, J. H. Wesenberg, R. B. Blakestad, R. J. Epstein, D. B. Hume, W. M. Itano, J. D. Jost, C. Langer, R. Ozeri, N. Shiga, and D. J. Wineland, Microfabricated Surface-Electrode Ion Trap for Scalable Quantum Information Processing, Phys. Rev. Lett. 96, 253003 (2006).

[5] G. De Chiara, A. del Campo, G. Morigi, M. B. Plenio, and A. Retzker, Spontaneous nucleation of structural defects in inhomogeneous ion chains, New J. Phys. 12, 115003 (2010).

[6] H. Landa, B. Reznik, J. Brox, M. Mielenz, and T. Schaetz, Structure, dynamics and bifurcations of discrete solitons in trapped ion crystals, New J. Phys. 15, 093003 (2013).

[7] M. Mielenz, J. Brox, S. Kahra, G. Leschhorn, M. Albert, T. Schaetz, H. Landa, and B. Reznik, Trapping of TopologicalStructural Defects in Coulomb Crystals, Phys. Rev. Lett. 110, 133004 (2013).

[8] H. L. Partner, R. Nigmatulin, T. Burgermeister, K. Pyka, J. Keller, A. Retzker, M. B. Plenio, and T. E. Mehlstaubler, Dynamics of topological defects in ion Coulomb crystals, New J. Phys. 15, 103013 (2013).

[9] K. Pyka, J. Keller, H. L. Partner, R. Nigmatulin, T. Burgermeister, D. M. Meier, K. Kuhlman, A. Retzker, M. B. Plenio, W. H. Zurek, A. del Campo, and T. E. Mehlstaubler, Topological defect formation and spontaneous symmetry breaking in ion Coulomb crystals, Nat. Commun. 4, 2291 (2013).

[10] S. Ulm, J. Rossnagel, G. Jacob, C. Degunther, S. T. Dawkins, U. G. Poschinger, R. Nigmatulin, A. Retzker, M. B. Plenio, F. Schmidt-Kaler, and K. Singer, Observation of the Kibble-Zurek scaling law for defect formation in ion crystals, Nat. Commun. 4, 2290 (2013).

[11] H. Landa, A. Retzker, T. Schaetz, and B. Reznik, Entanglement Generation Using Discrete Solitons in Coulomb Crystals, Phys. Rev. Lett. 113, 053001 (2014).

[12] H. L. Partner, R. Nigmatulin, T. Burgermeister, J. Keller, K. Pyka, M. B. Plenio, A. Retzker, W. H. Zurek, A. del Campo, and T. E. Mehlstaubler, Structural phase transitions and topological defects in ion Coulomb crystals, Physica B 460, 114 (2015).

[13] T. Dessup, T. Maimbourg, C. Coste, and M. Saint Jean, Linear instability of a zigzag pattern, Phys. Rev. E 91, 022908 (2015).

[14] G. Piacente, I. V. Schweigert, J. J. Betouras, and F. M. Peeters, Generic properties of a quasi-one-dimensional classical Wigner crystal, Phys. Rev. B 69, 045324 (2004).
[15] G. Piacente, G. Q. Hai, and F. M. Peeters, Continuous structural transitions in quasi-one-dimensional classical Wigner crystals, Phys. Rev. B 81, 024108 (2010).

[16] A. V. Straube, R. P. A. Dullens, L. Schimansky-Geier, and A. A. Louis, Zigzag transitions and nonequilibrium pattern formation in colloidal chains, J. Chem. Phys. 139, 134908 (2013).

[17] B. Liu and J. Goree, Phonons in a one-dimensional Yukawa chain: Dusty plasma experiment and model, Phys. Rev. E 71, 046410 (2005).

[18] A. Melzer, Zigzag transition of finite dust clusters, Phys. Rev. E 73, 056404 (2006).

[19] T. E. Sheridan, Dusty plasma ring model, Phys. Scr. 80, 065502 (2009).

[20] T. E. Sheridan and K. D. Wells, Dimensional phase transition in small Yukawa clusters, Phys. Rev. E 81, 016404 (2010).

[21] T. E. Sheridan and A. L. Magyar, Power law behavior for the zigzag transition in a Yukawa cluster, Phys. Plasmas 17, 113703 (2010).

[22] J.-B. Delfau, C. Coste, and M. Saint Jean, Transverse singlefile-diffusion near the zigzag transition, Phys. Rev. E 87, 032163 (2013).

[23] J.-B. Delfau, C. Coste, and M. Saint Jean, Noisy zigzag transition, fluctuations, and thermal bifurcation threshold, Phys. Rev. E 87, 062135 (2013).

[24] T. Dessup, C. Coste, and M. Saint Jean, Subcriticality of the zigzag transition: A nonlinear bifurcation analysis.Phys. Rev. E 91, 032917 (2015).

[25] T. Dessup, C. Coste, and M. Saint Jean, Hysteretic and intermittent regimes in the subcritical bifurcation of a quasione-dimensional system of interacting particles, Phys. Rev. E 93, 012105 (2016)

[26] O. M. Braun and Y. S. Kivshar, Nonlinear dynamics of the Frenkel-Kontorova model, Phys. Rep. 306, 1 (1998).

[27] M. Peyrard and T. Dauxois, Physique des Solitons (EDP Sciences and CNRS Editions, Les Ulis, 2004).

[28] H. Goldstein, Classical Mechanics (Addison Wesley, Reading, 1980).

[29] C. Coste, J.-B. Delfau, C. Even, and M. Saint Jean, Single file diffusion of macroscopic charged particles, Phys. Rev. E 81, 051201 (2010).

[30] J.-B. Delfau, C. Coste, and M. Saint Jean, Single file diffusion of particles with long-ranged interactions: Damping and finite size effects, Phys. Rev. E 84, 011101 (2011).

[31] J.-B. Delfau, C. Coste, C. Even, and M. Saint Jean, Single-file diffusion of interacting particles in a finite-sized channel, Phys. Rev. E 82, 031201 (2010).

[32] W. Dieterich, I. Peschel, and W. R. Schneider, Diffusion in periodic potentials, Z. Phys. B 27, 177 (1977).

[33] H. Risken, The Fokker-Planck Equation (Springer, Berlin, 1989).

[34] P. Reimann, C. Van den Broeck, H. Linke, P. Hänggi, J. M. Rubi, and A. Pérez-Madrid, Giant Acceleration of Free Diffusion by Use of Tilted Periodic Potentials, Phys. Rev. Lett. 87, 010602 (2001). 